\title{
Propriedades Mecânicas e de Inflamabilidade de Composições de Borracha EPDM Carregadas com Negro de Fumo e Hidróxido de Alumínio
}

\author{
Cristine Canaud, Leila L. Y. Visconte, Regina C. R. Nunes \\ Instituto de Macromoléculas Professora Eloisa Mano, UFRJ.
}

Resumo: Composições de EPDM (terpolímero de etileno-propileno-dieno) contendo misturas de negro de fumo e hidróxido de alumínio (ATH) foram preparadas com o objetivo de se avaliar seu potencial de utilização em aplicações elétricas. O hidróxido de alumínio, conhecido por suas características de retardante de chama, é uma carga semi-reforçante e, portanto, incapaz de fornecer o nível de reforço necessário para diversas aplicações. Dessa forma, para aliar ao artefato propriedades de não inflamabilidade e alto desempenho mecânico, foi também adicionado o negro de fumo (que é a carga reforçante mais largamente empregada). A escolha do EPDM se justifica pela facilidade de ser encontrado no mercado brasileiro, por sua característica em aceitar grandes quantidades de carga e por ser um elastômero apolar, o que é um requisito básico para aplicação em isolamento-elétrico. Os materiais foram submetidos a testes mecânicos e de inflamabilidade, para se determinar aqueles que se ajustavam às normas estabelecidas pela Associação Brasileira de Normas Técnicas - ABNT. Verificou-se que pelo menos 160 phr de hidróxido de alumínio são necessários nessas composições para que a resistência à chama seja alcançada.

Palavras-chave: Elastômero EPDM, hidróxido de alumínio, propriedades mecânicas, resistência à chama.

\section{Mechanical and flammability properties of EPDM (ethylene-propylene terpolymer) rubber compositions filled with carbon black and aluminum hydroxide}

\begin{abstract}
EPDM composition filled with mixtures of aluminum hydroxide (ATH) and carbon black were evaluated for electrical applications. Aluminum hydroxide, known for its characteristics of flame retardancy, is a semi-reinforcing filler and, in this sense, it is unable to impart the reinforcement level necessary to several applications. Thus, to have in the same artifact the non-flammability properties together with a high mechanical performance, carbon black, the most widely used reinforcing filler, has also been used. The choice of EPDM can be justified by the availability of this polymer in the brazilian market, allied to its characteristics to accept large amounts of filler and its apolar chemical character, which is a basic requirement for application in electrical insulation. To meet the Brazilian Technical Standards - ABNT, mechanical and flammability tests were carried out in order to find out the relative amounts of the two fillers which would give the best balance of properties. Amounts as high as $160 \mathrm{phr}$ of aluminum hydroxide are required to achieve good flame resistance.
\end{abstract}

Keywords: EPDM compositions, aluminum hydroxide, flammability, mechanical properties.

\section{Introdução}

As propriedades mecânicas de sistemas poliméricos estão entre as mais importantes quando se consideram determinadas aplicações. Isso deve ser creditado ao fato de que, para qualquer condição real de trabalho, propriedades como resistência à tração, módulo, alongamento na ruptura e resistência ao impacto se fazem necessárias na seleção de um material polimérico ${ }^{[1-7]}$. Uma fonte de informações sobre o comportamento de

Autor para correspondência: Regina C. R. Nunes, Instituto de Macromoléculas Professora Eloisa Mano, UFRJ, C. P. 68525, Cidade Universitária, Ilha do Fundão, Centro de Tecnologia, Bloco J, CEP: 21945-970 Rio de Janeiro, RJ, E-mail: rcnunes@ima.ufrj.br 
materiais é o estudo das variações das propriedades durante o processo de envelhecimento. Esse tipo de estudo tem sido feito com relação a propriedades mecânicas de várias borrachas reforçadas mas, com menor freqüência no caso de composições com EPDM ${ }^{[8-11]}$, o que pode ser justificado pela estrutura molecular deste elastômero. O conjunto de propriedades, como excelente resistência à degradação, boa resistência química e excelentes propriedades elétricas ${ }^{[12-15]}$ tem incentivado o uso desse elastômero em composições para isoladores elétricos, em fios e cabos para baixa e média voltagem (até $35 \mathrm{kV}$ ) ${ }^{[12,13,16-18]}$. No caso de aplicações externas, os materiais devem apresentar propriedades elétricas e resistência mecânica para suportar ataques físicos e estabilidade contra degradação ambiental.

O envelhecimento em materiais poliméricos pode ser provocado por oxidação, exposição a raios UV, ou por uma perda gradual de plastificante ou outros aditivos de baixo peso molecular ${ }^{[19-27]}$. O efeito do envelhecimento nas propriedades elétricas de polímeros reforçados com negro de fumo é muito pequeno à temperaturas ordinárias mas a altas temperaturas o efeito sobre a condução elétrica pode ser considerável em plásticos e borrachas ${ }^{[22,26,28-32]}$.

Devido à utilização cada vez maior de materiais poliméricos em estruturas e artefatos em geral, há uma grande preocupação com o perigo à vida humana por causa das possibilidades de incêndio, como a liberação de fumaças e gases tóxicos. Esses materiais ao serem queimados geram produtos que agem como combustível, de modo que o retardante de chama tem o propósito de aumentar a resistência desses materiais à ignição e, ao mesmo tempo, reduzir a velocidade de propagação da chama.

Em geral, os testes de inflamabilidade podem ser classificados como sendo de pequena escala e de grande escala. Nos testes em grande escala procura-se fazer uma simulação que se aproxime o máximo possível das condições reais de trabalho. São testes caros que demandam longos períodos de tempo e, portanto, difíceis de serem realizados em laboratório, para controle do material. Testes feitos em pequena escala não simulam incêndios mas ainda assim são úteis como uma medida do efeito de determinados parâmetros sobre o comportamento do material frente o incêndio. Os resultados, dependendo do teste em particular, podem apresentar valores numéri- cos ou ser do tipo classificatório ${ }^{[33,34]}$.

Na indústria elétrica, a avaliação da inflamabilidade fornece dados comparativos em relação à resistência à chama, sob condições específicas, e é geralmente aplicada durante o estágio de desenvolvimento do produto e controle de qualidade ${ }^{[35]}$. Entre esses testes pode-se citar o Underwriters Laboratories 94 (UL 94) ${ }^{[36]}$.

Uma das maneiras encontradas para se preparar materiais com retardante de chamas é a incorporação de aditivos que durante a queima sejam decompostos, absorvendo energia da fonte de ignição e liberando vapor d'água. O hidróxido de alumínio (ATH) é o agente retardante de chama mais utilizado e o seu consumo corresponde a $50 \%$ do volume total de todos os retardantes de chama consumidos no mundo. As principais vantagens do ATH são o baixo custo e a baixa toxidez, decorrente da não liberação de gases tóxicos ou substâncias corrosivas durante a queima, agindo simultaneamente como retardante de chama e supressor de fumaça ${ }^{[37]}$.

No entanto, com relação ao desempenho mecânico, o ATH tem apenas caráter semi-reforçante e a sua presença não provoca melhoras significativas na resistência mecânica das misturas. Para contornar esta limitação, ATH com tratamento superficial com silano foi testado em combinação com negro de fumo, a carga mais usada em elastômeros. Neste trabalho, misturas contendo diferentes quantidades de ATH e negro de fumo foram incorporadas à borracha EPDM com o objetivo de se encontrar um balanço adequado de propriedades para utilização como revestimento isolante com características de resistência à chama, para fios e cabos elétricos. As composições foram avaliadas quanto aos desempenhos mecânico e de inflamabilidade, antes e após o envelhecimento.

\section{Experimental}

A Tabela 1 apresenta as formulações usadas na preparação das composições. Os ingredientes, de grau comercial foram adicionados a um misturador de cilindros, à temperatura ambiente e de acordo com a norma ASTM D3568. Os parâmetros de vulcanização foram obtidos em reômetro de disco oscilatório da marca Monsanto, a $160^{\circ} \mathrm{C}$, mesma temperatura em que foi realizada a vulcanização das composições. $\mathrm{O}$ envelhecimento foi feito a $100^{\circ} \mathrm{C}$ durante 7 dias em estufa com circulação forçada de ar.

As composições serão designadas pela razão en- 
Tabela 1. Formulações usadas nas composições de EPDM (as quantidades são dadas em phr)

\begin{tabular}{|c|c|c|c|c|c|c|c|}
\hline Ingrediente & 1 & 2 & 3 & 4 & 5 & 6 & 7 \\
\hline EPDM 57C & 100 & 100 & 100 & 100 & 100 & 100 & 100 \\
\hline $\begin{array}{l}\text { Negro de } \\
\text { fumo } N 330^{b}\end{array}$ & 45 & 30 & 22 & 15 & 7.5 & 0 & 0 \\
\hline $\mathrm{ATH}^{\mathrm{c}}$ & 120 & 140 & 150 & 160 & 170 & 180 & 0 \\
\hline $\begin{array}{l}\text { Óxido de } \\
\text { zinco }\end{array}$ & 5 & 5 & 5 & 5 & 5 & 5 & 5 \\
\hline $\begin{array}{l}\text { Ácido } \\
\text { esteárico }\end{array}$ & 1 & 1 & 1 & 1 & 1 & 1 & 1 \\
\hline Enxofre & 1.5 & 1.5 & 1.5 & 1.5 & 1.5 & 1.5 & 1.5 \\
\hline $\begin{array}{l}\text { Óleo } \\
\text { parafínico }\end{array}$ & 25 & 25 & 25 & 25 & 25 & 25 & 25 \\
\hline $\mathrm{MBT}^{\mathrm{d}}$ & 0.5 & 0.5 & 0.5 & 0.5 & 0.5 & 0.5 & 0.5 \\
\hline TMTD $^{\mathrm{e}}$ & 1.0 & 1.0 & 1.0 & 1.0 & 1.0 & 1.0 & 1.0 \\
\hline
\end{tabular}

a Procedência: DSM do Brasil Ltda; razão etileno/propileno: 73/27; índice de iodo: 15; viscosidade Mooney: $90 \mathrm{ML}_{1+4\left(100^{\circ} \mathrm{C}\right.}$

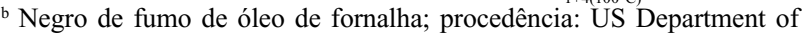
Commerce National Bureau of Standards; densidade: $1,80 \mathrm{~g} / \mathrm{cm}^{3}$

c Hidróxido de alumínio; 2 tipos foram usados: tratado e não tratado com silano

${ }^{d}$ Mercaptobenzotiazol

e Dissulfeto de tetrametiltiuram

tre as cargas ATH/negro de fumo, em phr. Por exemplo, a composição 150/22 é aquela contendo $150 \mathrm{phr}$ de ATH e 22 phr de negro de fumo.

\section{Resultados e Discussão}

É apresentado na Figura 1 um histograma com os valores de resistência à tração para as composições com diferentes razões ATH/N330, antes e após o envelhecimento.

A partir dos dados da Figura 1, o caráter reforçante do negro de fumo pode ser visto para aquelas composições com as quantidades mais altas desta carga. Por outro lado, a sua ausência na composição 180/0 confirma o caráter semi-reforçante do ATH, pois o valor desta propriedade para esta composição só é maior quando comparado com a goma pura (EPDM contendo todos os ingredientes à exceção da carga). Aumentando-se o conteúdo de ATH também torna as composições mais sensíveis ao envelhecimento. Nessas composições esses efeitos são menos pronunciados quando o ATH é tratado com silano,

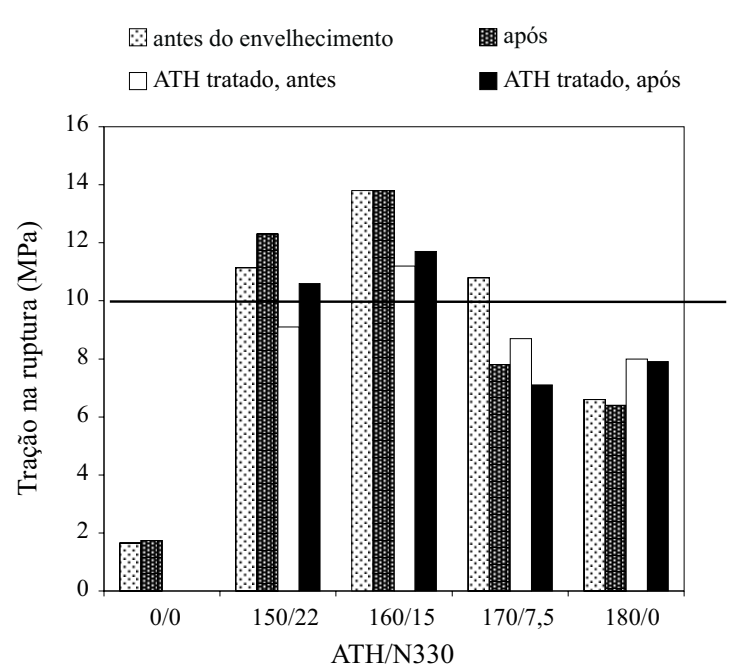

Figura 1. Tração na ruptura das composições de EPDM, antes e após envelhecimento

evidenciando a importância do tratamento superficial. O ATH tratado diminui a resistência à tração quando comparado com os resultados para o ATH sem tratamento, com exceção da composição sem negro de fumo.

A Associação Brasileira de Normas Técnicas, $\mathrm{ABNT}$, exige que os materiais usados em fios e cabos, submetidos a baixa e média voltagens, estejam de acordo com as seguintes especificações: a tração na ruptura deve ser pelo menos igual a $10 \mathrm{MPa}$, antes do envelhecimento, com uma variação máxima de $\pm 30 \%$, após o envelhecimento.

De acordo com a Figura 1, as composições que se ajustam às exigências da ABNT são: 150/22 e 160/15 (ATH com e sem tratamento) e 170/7,5.

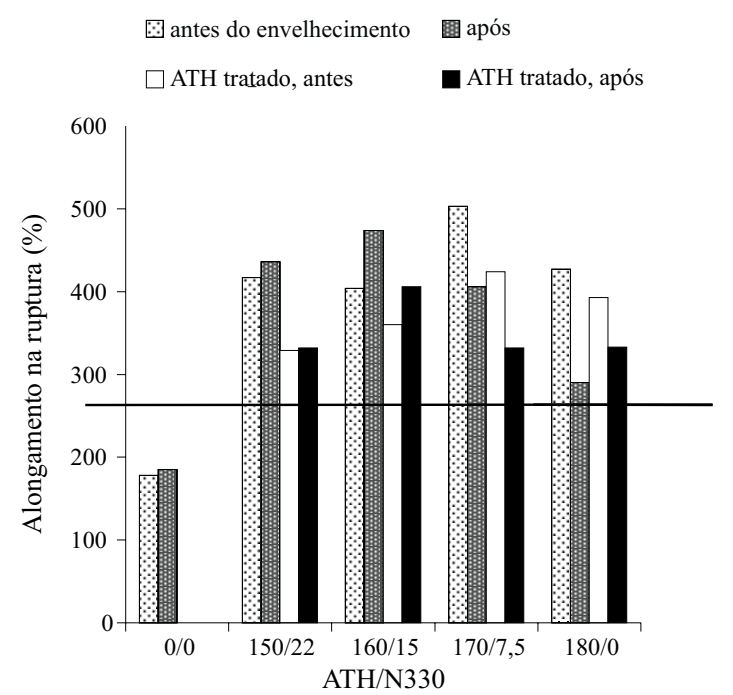

Figura 2. Alongamento na ruptura para composições de EPDM, antes e após envelhecimento 


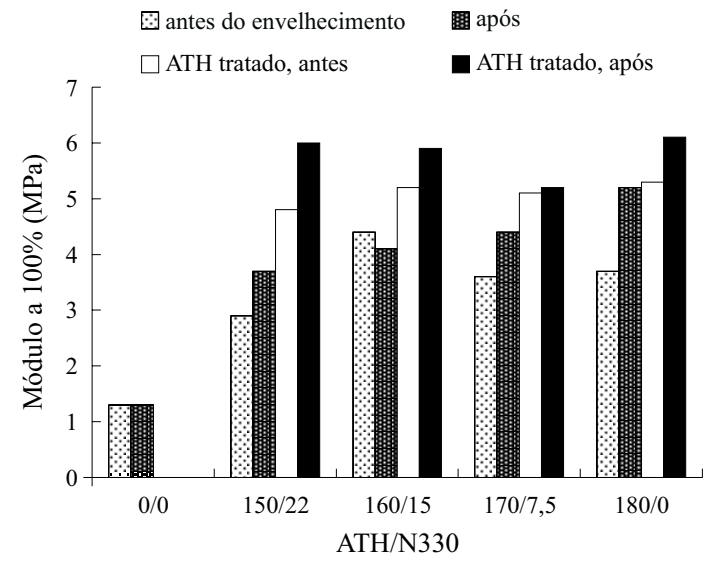

Figura 3. Módulo a $100 \%$ de composições de EPDM, em função da razão $\mathrm{ATH} / \mathrm{N} 330$

Dados de alongamento, antes e após o envelhecimento podem ser vistos na Figura 2.

De acordo com a ABNT, para uso em fios e cabos elétricos, os materiais devem apresentar pelo menos $300 \%$ de alongamento e uma variação máxima de $\pm 40 \%$ sobre o valor original, após o envelhecimento. Dos materiais testados, conforme pode ser visto na Figura 2, todos estão em conformidade com a norma, com exceção da goma pura (composição com todos os aditivos, exceto a carga), sendo que as composições em que o ATH sofreu tratamento superficial são menos sensíveis à degradação.

Valores de módulo a $100 \%$ podem ser vistos na Figura 3.

Os dados mostram que, com relação a esta propriedade, todas as formulações são superiores à goma pura (composição sem carga), principalmente aquelas com ATH tratado, muito embora não apresentem grandes variações nos valores em função da quanti-

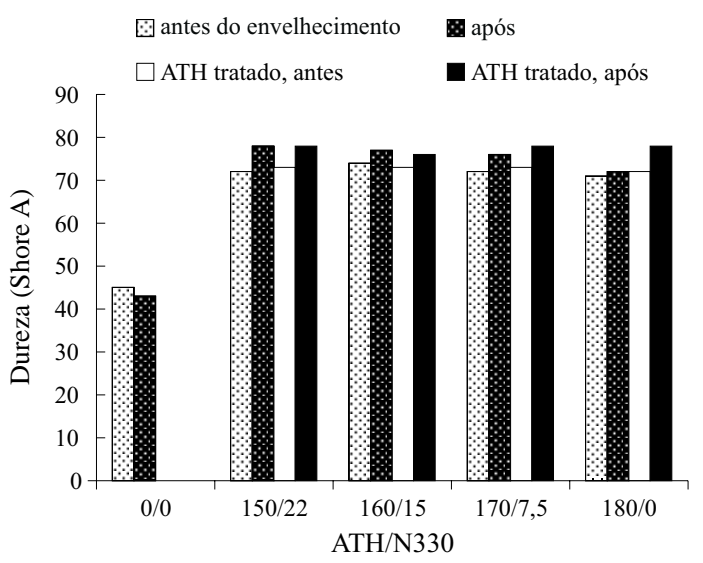

Figura 4. Influência da razão ATH/N330 sobre a dureza de composições de EPDM, antes e após envelhecimento dade de ATH, em ambas as séries. Após o envelhecimento o mesmo comportamento é encontrado e os valores de módulo são superiores aos obtidos antes do envelhecimento. Isto provavelmente se deve a um aumento no número de ligações cruzadas que são formadas durante o processo de envelhecimento, o que pode justificar, de maneira geral, o aumento da dureza nas composições após envelhecimento.

A Figura 4 mostra dados de dureza Shore em função da composição da carga.

A dureza mede a resistência que um material apresenta quando submetido a uma deformação local ${ }^{[1-3]}$, e depende, entre outros fatores, da quantidade de carga e do número de ligações cruzadas, podendo ser relacionada com o módulo. Assim, da mesma maneira que o módulo, também a dureza é aumentada pela incorporação de cargas, como mostrado na Figura 4, já que todas as composições apresentaram valores de dureza maiores que a goma pura. $\mathrm{O}$ método não foi sensível ao tipo ou à quantidade de $\mathrm{ATH}$; no entanto, após o envelhecimento, como comentado anteriormente, houve um incremento pouco significativo nesses sugerindo a formação de ligações cruzadas durante $o$

Tabela 2. Resultados do teste de inflamabilidade UL 94 para composições de EPDM

\begin{tabular}{|c|c|}
\hline ATH/HAF (phr) & Classificação \\
\hline $0 / 0$ & $\mathrm{~V} 2$ \\
\hline $120 / 45^{*}$ & $\mathrm{~V} 2$ \\
\hline $120 / 45$ & $\mathrm{~V} 2$ \\
\hline $140 / 30 *$ & $\mathrm{~V} 2$ \\
\hline $140 / 30$ & $\mathrm{~V} 2$ \\
\hline $150 / 22 *$ & $\mathrm{~V} 2$ \\
\hline $150 / 22$ & $\mathrm{~V} 2$ \\
\hline $160 / 15^{*}$ & V0 \\
\hline $160 / 15$ & V0 \\
\hline $170 / 7.5^{*}$ & V0 \\
\hline $170 / 7.5$ & V0 \\
\hline $180 / 0 *$ & V0 \\
\hline $180 / 0$ & V0 \\
\hline
\end{tabular}

\footnotetext{
*ATH tratado com silano
} 
Tabela 3. Classificação dos materiais segundo a norma UL 94[36]

\begin{tabular}{|c|c|c|c|}
\hline Critérios & Vo & V1 & $\mathbf{V 2}$ \\
\hline $\begin{array}{l}\text { Valores individuais de } \mathrm{t} 1 \\
\text { ou t } 2\end{array}$ & $\leq 10 \mathrm{~s}$ & $\leq 30 \mathrm{~s}$ & $\leq 30 \mathrm{~s}$ \\
\hline $\begin{array}{l}\text { Somatórios de }(\mathrm{t} 1+\mathrm{t} 2) \\
\text { para os corpos de prova }\end{array}$ & $\leq 50 \mathrm{~s}$ & $\leq 250 \mathrm{~s}$ & $\leq 250 \mathrm{~s}$ \\
\hline Valores individuais de $\mathrm{t} 3$ & $\leq 30 \mathrm{~s}$ & $\leq 60 \mathrm{~s}$ & $\leq 60 \mathrm{~s}$ \\
\hline $\begin{array}{l}\text { Queima com chama ou } \\
\text { incandescência até o } \\
\text { prendedor }\end{array}$ & não & não & não \\
\hline $\begin{array}{l}\text { Queima do algodão por } \\
\text { gotas ou fagulhas emitida }\end{array}$ & não & não & $\operatorname{sim}$ \\
\hline
\end{tabular}

$\mathrm{t}_{1}$ : tempo de duração da chama no corpo de prova após a primeira aplicação, em minutos;

$\mathrm{t}_{2}$ : tempo de duração da chama no corpo de prova após a segunda aplicação, em minutos;

$\mathrm{t}_{3}$ : tempo de duração da chama mais incandescência após a segunda aplicação, em minutos.

processo.

O teste utilizado para a classificação dos materiais quanto à inflamabilidade foi desenvolvido por Underwriters Laboratories (UL 94) e forneceu os resultados apresentados na Tabela 2. Este teste classifica os materiais de acordo com os critérios ${ }^{[36]}$ da Tabela 3.

De acordo com os resultados da Tabela 2, as composições contendo ATH em quantidades maiores que $160 \mathrm{phr}$, com ou sem tratamento superficial, alcançaram o índice de propagação V-0. No entanto, ao se considerar também as exigências ABNT quanto às propriedades mecânicas, somente as formulações 160/ 15 (ATH com e sem tratamento) e 170/7,5 podem ser consideradas adequadas para aplicação em fios e cabos para voltagens até $15 \mathrm{kV}$.

\section{Conclusões}

De acordo com a ABNT, os materiais poliméricos para uso em fios e cabos elétricos em voltagens de até $15 \mathrm{kV}$ devem apresentar as seguintes propriedades mecânicas:

- resistência à tração mínima de $10 \mathrm{MPa}$, admitindo-se uma variação máxima de $\exists 0 \%$, após o envelhecimento;

- alongamento de pelo menos $300 \%$ e variação máxima de $\pm 40 \%$ sobre esse valor, após o envelhecimento.

Dos materiais investigados, as composições 150/
22 (contendo ATH sem tratamento), 160/15 (contendo ATH com ou sem tratamento) e 170/7,5 (contendo ATH sem tratamento), ajustam-se às exigências da ABNT, de acordo com os critérios acima relacionados.

Entretanto, levando-se em conta também as características de inflamabilidade desses materiais, apenas as composições 160/15(contendo ATH com ou sem tratamento) e 170/7,5 (com ATH sem tratamento) alcançam a classificação V0 e podem ser consideradas dentro dos padrões exigidos para aplicação em fios e cabos elétricos, sob voltagens de até $15 \mathrm{kV}$.

\section{Agradecimentos}

Os autores agradecem à Alcoa Alumínio S. A., pelo apoio financeiro.

\section{Referências Bibliográficas}

1. Sheldon, R. P. - "Mechanical properties of composites", in: Composite Polymeric Materials, Sheldon, R. P. (ed), Applied Science Publishers, New York, p.58 (1982).

2. Shah, V. - "Mechanical properties", in: Handbook of Plastics Testing Technology, Shah, R. P. (ed), John Wiley \& Sons, New York, p.7 (1984).

3. Seymor, R. B. - "Properties of polymers", in: Properties of Solids, Seymor, R. B. (ed), , ASM International, USA, p.17 (1987).

4. Freakly, P. K. \& Payne, A. R., "Theory and Practice of Engineering with Rubber", Applied Science Publishers, New York, (1978).

5. Sommer, J. G. - Rubber World, 214, p.15 (1996).

6. Seldon R. P. - "Composite Polymeric Materials", Applied Science Publishers, New York (1982).

7. Gent, N. A. - "Strength of elastomers", in: Science and Technology of Rubber, Mark, J. E.; Erman, B. \& Eirich, F. R. (ed), Academic Press, New York, p. 471 (1994).

8. C. R. Frihart, R. L. Gordon - Adhesive Age, 39, p.20 (1996).

9. G. S. Keidiya, E. M. Ermenko, V. M. Aristov, Y. V. Zelenev - International Polymer Science and Technology 24, p.87 (1997).

10. G. M. Nasr, A. S. Gomaa - Polymer Degradation and Stability 50, p.249 (1995).

11. R. L. Clough - Journal of Polymer Science 21, p.767 
(1983).

12. Cheremisinoff, N. P. - "Properties and uses of ethylene-propylene rubbers", in: Handbook of Polymer Science and Technology, Cheremisinoff, N. P. (ed), Marcel Dekker, New York, p. 113 (1989).

13. Strate, G. V. - "Ethylene-propylene elastomers", in: Encyclopedia of Polymer Science and Technology, Mark, H.; Bikales, N. M \&. Menges, C. G (ed), John Wiley \& Sons, New York, p.523 (1986).

14. Schowob, Y. - Caoutchoucs \& Plastiques, 733, p.72 (1994)

15. Arnol, L. K. - "Ethylene rubber", in: Introduction to Plastics, Allen, G. (ed), George Allen and Unwin Ltd, London, p.151 (1969).

16. Allen, R. D. - "Improving the high-temperature performance of EPDM", in: Handbook of Polymer Science and Technology, Cheremisinoff, N. P. (ed), Marcel Dekker, New York, p.127 (1989).

17. Keeley, F. W. \& Vaidya, U. I. - "Nordel in wire and cable electrical elastomers", Du Pont, 6 (1956).

18. Jones, F. K.; Laird, J. L. \& Smith, B. W. - Rubber World, 215, p.42 (1996).

19. Guzzo, M. \& De Paoli, M. A. - Polymer Degradation and Stability, 38, p.41 (1992).

20. Guzzo, M. \& De Paoli, M. A. - Polymer Degradation and Stabilization, 36, p.169 (1992).

21. Kole, S.; Chaki, Y. K; Bhowmick, A. K. \& Tripathy, D. K. - Polymer Degradation and Stability, 47, p.397 (1996).

22. Gueguen, V.; Audouin, L.; Pinel, B. \& Verdu, J. - Polymer Degradation and Stability, 46, p.113 (1994).

23. Teissedre, G.; Pilichowski, J. F.; Chmela, S. \& Lacoste, J. - Polymer Degradation and Stability,
53, p.207 (1996).

24. Gueguen, V.; Audoun, L.; Pinel, B. \& Verdu, J. - Polymer Degradation and Stability, 43, p.217 (1994).

25. Giurginca, M.; Zahaarescu, T. \& Meghea, A. _ Polymer Degradation and Stability, 50, p.45 (1995).

26. Scott, G. - Polymer Degradation and Stability, 48, p.315 (1995).

27. Ehrhardt, D. - International Polymer Science and Technology, 24, p.14 (1997).

28. Keidiya, G. S.; Ermenko, E. M.; Aristov, V. M. \& Zelenev, Y. V. - International Polymer Science and Technology, 24, p.87 (1997).

29. Nasr, G. M. \& Gomaa, A. S. - Polymer Degradation and Stability, 50, p.249 (1995).

30. Chailan, J. F.; Boiteux, G.; Chauuchard, J.; Pinel, B. \& Seytre, G. - Polymer Degradation and Stability, 47, p.397 (1995).

31. Gallagher, M. T. - Rubber World, 211, p.26 (1994).

32. Aziz, M. M. A.; Gwaily, S. E.; Makarious, A. S. \& A. S. Abdo, A. S. - Polymer Degradation and Stability, 50, p.235 (1995).

33. Hirschler, M. M. - Polymer Degradation and Stabilization, 54, p.333 (1996).

34. Schulz, N. - Rubber World, 213, p.18 (1996).

35. Eliott, A. N. A. - "Processing, bonding, fire retardants", Rapra Reviews Reports, 6, p.3 (1997).

36. "Tests for flammability of plastic materials for parts in devices and appliances", Underwriters Laboratories Inc., UL94, USA, 1991.

37. Sobolev, A. I. \& Wasycheshim, E. A. - "Alumina Trihydrate", in: "Handbook of Fillers for Plastics", Katz, H. S. \& Milewski, I. V. (ed), Van Nostrand Reinhold, New York, 1987, cap.5, pp. 292-301. 